\title{
Characteristics of primary side population cervical cancer cells
}

\author{
ZHEN-TONG WEI $^{1 *}$, XIAO-WEI YU ${ }^{2 *}$, JIA-XUE HE ${ }^{3}$, YAN LIU $^{4}$ and SONG-LING ZHANG ${ }^{1}$ \\ ${ }^{1}$ Department of Gynecological Oncology; ${ }^{2}$ Prenatal Diagnosis Center; ${ }^{3}$ Genetic Diagnosis Center, \\ The First Hospital of Jilin University, Changchun, Jilin 130021; ${ }^{4}$ Genetic Engineering Laboratory of The Chinese People's \\ Liberation Army, Military Veterinary Institute, Acadamy of Military Medical Sciences, Changchun, Jilin 130122, P.R. China
}

Received June 30, 2015; Accepted March 9, 2017

DOI: $10.3892 / \mathrm{ol} .2017 .6606$

\begin{abstract}
The aim of the present study was to identify and characterize side population (SP) cells in primary cervical cancer. A primary culture was successfully established, and the SP cells were isolated via fluorescence-activated cell sorting. Subsequently, in vitro analysis of clonogenic capacity by soft agar assay and in vivo analysis of tumorigenicity were performed. The isolated SP cells accounted for $\sim 4.73 \%$ of the total primary culture cells. The SP cells had a decreased proliferation rate and an increased distribution in $\mathrm{G}_{0} / \mathrm{G}_{1}$ compared with non-SP (NSP) cells. Following isolation, SP cells exhibited increased proliferative and self-renewal potency compared with NSP cells. Furthermore, significant ATP binding cassette subfamily G member 2 (ABCG2) expression was detected in SP cells but not in NSP cells. The tumor formation rate of SP cells was longer, and the tumor size and tumor formation rate of SP cells were increased in non-obese diabetic/severe combined immunodeficiency mice. In conclusion, the present study demonstrated that SP cells can be isolated from primary cervical cancer cell culture, and SP cells are enriched with stem cell-like cells that have a high capacity for colony formation and tumorigenesis.
\end{abstract}

\section{Introduction}

According to the stem cell theory of cancer, a small number of tumors cells present in primary tumors and act as stem cells with unlimited proliferative potential; these cells have a crucial role in driving tumor formation, growth, relapse and metastasis (1). Normal somatic stem cells are naturally resistant to chemotherapeutic agents, as these cells can pump out drugs through various multidrug resistance (MDR) pumps

Correspondence to: Dr Song-Ling Zhang, Department of Gynecological Oncology, The First Hospital of Jilin University, 71 Xinmin Avenue, Changchun, Jilin 130021, P.R. China

E-mail: slzhang@jlu.edu.cn

*Contributed equally

Key words: cervical cancer, cancer stem cells, primary culture, SP cells, soft agar, xenograft and have a slow rate of cell turnover (chemotherapeutic agents target rapidly replicating cells) (2).

Cancer stem cells (CSCs), which derive from normal stem cells, also produce MDR proteins and therefore increase their resistance towards chemotherapeutic agents (3). The CSCs that resist chemotherapy survive and subsequently repopulate the cancerous area; consequently, this causes a relapse. Therapies that selectively target CSCs enable the treatment of patients with non-resectable aggressive tumors and may therefore prevent metastases (3). It has been suggested that when CSCs are eliminated, complete tumor regression can be achieved with residual cells, which display a differentiated phenotype and are susceptible to apoptosis (1). Therefore, CSCs-targeted therapy has been proposed to be a promising cancer treatment approach, particularly for certain types of cancer cases that occur in the uterus and cervix.

Cancer stem cells from acute myeloid leukemia (4), breast cancer (5), glioma (6) and a number of other types of tumors have been successfully isolated using cancer specific cell surface markers. However, due to the lack of specific surface markers, CSCs have not been isolated from many other types of cancer, particularly from solid tumors including cervical cancer. In 1996, Goodell et al (7) identified a subset of cells with low Hoechst 33342 staining from murine bone marrow. It was demonstrated that these cells exhibit hematopoietic stem cell features and were able to be identified as a side population (SP) in flow cytometric assays. Since then, SP cell sorting has been used to isolate stem cells from tissues without the need for specific stem cell surface markers. For example, Kondo et al (8) isolated SP cells from the C6 tumor cell line and confirmed the multi-differentiation potencies and the tumorigenicity of these SP cells. This suggests that SP cell sorting may be applied to the isolation of CSCs from cancer cell lines. Additionally, Patrawala et al (9) provided evidence for the presence of SP cells in 9/30 tumors cell lines, including cell lines established from melanoma and prostate, breast, colon, glioma, bladder, ovarian, cervical and nasopharyngeal cancer. The percentage of SP cells in these cell lines ranged between 0.04 and $0.2 \%$ of the overall cell population. These results indicated that SP cells only accounted for a small proportion of the total cell population and may only be detected in a number of human tumor cell lines. Additionally, two studies have reported the presence of $\sim 1 \%$ SP cells in the HeLa cell line $(10,11)$.

To the best of our knowledge, the isolation and characterization of SP cells from primary cervical cancer cell cultures 
has not been reported. However, these well-established cell lines may have gone through extensive genomic changes and therefore may not represent in vivo tissues as closely as primary cell cultures. In the present study, SP cells were successfully isolated from a primary cervical cancer cell culture. In vitro and in vivo assays validated the stem cell features of these SP cells.

\section{Materials and methods}

Ethics. The present study was conducted in accordance with international guidelines and approved by the Ethics Committee of The First Hospital of Jilin University (Changchun, China). Written informed consent was obtained from the patients. All efforts were made to minimize suffering by conducting procedures according to Animal Care Guidelines. The animal experiment was approved by the Ethics Committee of The First Hospital of Jilin University (Changchun, China).

Establishment of primary cervical cancer cell culture. Between December 2011 and June 2012, the surgical specimens were collected from 10 female patients with cervical squamous cell carcinoma at stage IB2 according to the staging system established by the International Federation of Gynecology and Obstetrics in 2009 (12). The age of patients ranged from 43-51 years. All patients were HPV positive and did not undergo preoperative chemotherapy. Primary cells, derived from 1 patient, were successfully cultured by explant culture method. Purified cervical cancer cells were harvested following repeated cycles of attachment and mechanical curettage that gradually leads to the elimination of fibroblasts. Cells were maintained in Dulbecco's modified Eagle's medium (DMEM), supplemented with $10 \%$ fetal bovine serum (FBS) (Gibco; Thermo Fisher Scientific, Inc., Waltham, MA, USA). Half of the culture medium was changed every 3-4 days until the cells grew to $80 \%$ confluence at which stage the culture was split for the first passage. Following 10 passages, the cells were split every 6-8 days (at a ratio of $1: 3$ ) by trypsinization. The nucleus-to-cytoplasm ratio was determined by the following equation: The nucleus-to-cytoplasm ratio $=($ The diameter of nucleus/The width of cytosol) $\mathrm{x} 100 \%$.

Animals. A total of 5 5-week-old female BALB/C nude mice (weighing 16-20 g) and 15 5-week-old female non-obese diabetic/severe combined immunodeficiency (NOD/SCID) mice (weighing 16-20 g) were purchased from Vital River Laboratories, Co., Ltd. (Beijing, China). Animals were housed in a sterilized room with $12 \mathrm{~h}$ light/dark cycle, at a temperature of $22^{\circ} \mathrm{C}$ with $40-60 \%$ humidity. Food and water were provided ad libitum.

Tumorigenicity assay for primary cervical cancer cells. The primary cancer cells in the exponential growth phase at passage 10 were harvested by trypsinization. The cells $\left(1 \times 10^{6}\right)$ were resuspended in a mixture of (200 $\mu \mathrm{l})$ DMEM/F12 (Gibco; Thermo Fisher Scientific, Inc.) and Matrigel (Sigma-Aldrich; Merck KGaA, Darmstadt, Germany) with a ratio of 1:1. The cells were subsequently subcutaneously inoculated into female BALB/C nude mice purchased from Vital River Laboratories,
Co., Ltd. (Beijing, China). The mice were examined daily, and the time points of tumor appearance were recorded.

Sample preparation and histological analysis. Mice were sacrificed 8 weeks following inoculation, and the tumor tissues were collected, weighed and fixed in $10 \%$ formalin for 3 days at room temperature. The tissues were sectioned into $3 \mathrm{~mm}$ thickness. Sections were stained with hematoxylin for $5 \mathrm{~min}$ at room temperature. Subsequent to washing with tap water, samples were stained with $0.5 \%$ eosin for 3 min at room temperature. After washing with tap water, samples were examined under phase contrast microscope (IX50; Olympus Corporation, Tokyo, Japan) for histological analysis.

Immunohistochemical analysis of transplantation tumors. Transplanted cervical carcinoma derived from nude mice were fixed in $10 \%$ formalin for 3 days at room temperature. The tissues were sliced into $3 \mathrm{~mm}$-thick sections. Samples were deparaffinized in xylene and dehydrated in an ethanol series, followed by washing three times with PBS for $5 \mathrm{~min}$. Samples were then exposed to heating in citrate buffer and the peroxidase was blocked for $15 \mathrm{~min}$ at room temperature. Subsequent to blocking in nonimmunone animal serum (Maxim Biotech, Inc., Rockville, MD, USA) for $30 \mathrm{~min}$ at room temperature, samples were stained with the cervical cancer-specific antibody p63 (EPR5701; catalog no., ab124762; Abcam, Cambridge, MA, USA; dilution, $1: 100$ ) at $4^{\circ} \mathrm{C}$ overnight. Subsequent to washing, samples were stained with biotin-conjugated secondary antibody (cat. no., KIT-9730; dilution, ready to use) for $30 \mathrm{~min}$ at room temperature, followed by $30 \mathrm{~min}$ incubation at room temperature with streptavidin-peroxidase using the UltraSensitive $\mathrm{S}-\mathrm{P}$ kit according to the manufacturer's protocol (Maxim Biotech, Inc.). Immunostaining was visualized by DAB reagent (Maxim Biotech, Inc.). The nuclei were stained with hematoxylin and samples were visualized under phase contrast microscope at magnification, x40 (IX50; Olympus Corporation, Tokyo, Japan). A total of five non-overlapped fields were randomly selected and images were analyzed by Image-Pro Plus 6.0 software (Media Cybernetics, Inc., Rockville, MD, USA).

Detection, sorting, and culture from primary cervical cancer cells by fluorescence-activated cell sorting (FACS). The primary cervical cancer cells in the exponential growth phase were harvested and re-suspended in warm DMEM containing $2 \%$ flow cytometry staining buffer (CycleTEST ${ }^{\mathrm{TM}}$ PLUS DNA Reagent kit; BD Biosciences) at a density of $1 \times 10^{6}$ cells $/ \mathrm{ml}$. The cell suspensions were stained with Hoechst $33342(5 \mu \mathrm{g} / \mathrm{ml})$ in the presence or absence of verapamil (ABC transporter blocker, $50 \mu \mathrm{mol} / 1$; Sigma-Aldrich; Merck KGaA) at $37^{\circ} \mathrm{C}$ for $90 \mathrm{~min}$ with intermittent mixing. Following incubation, the cells were washed once and re-suspended in ice-cold PBS containing $2 \%$ FBS (2\% FBS/PBS) (Gibco; Thermo Fisher Scientific, Inc., Waltham, MA, USA) and 2 mM 4-(2-hydroxyethyl)-1-piperazineethanesulphonic acid buffer. Following centrifugation at $380 \mathrm{x} \mathrm{g}$ for $6 \mathrm{~min}$ at $4^{\circ} \mathrm{C}$, supernatant was removed, and samples were washed and resuspended with $2 \% \mathrm{FBS} / \mathrm{PBS}$. Prior to analysis and sorting by flow cytometry using FACS Influx (BD Biosciences, Franklin Lakes, NJ, USA), the cells were filtered through a filter (pore size, $37-\mu \mathrm{m}$ ) and stained 
with propidium iodide (PI, $2 \mu \mathrm{g} / \mathrm{ml}$ ) for 15 min to determine cell viability. Cells were rinsed with $2 \%$ FBS/PBS. Sorted SP and non-SP (NSP) cells ( $1 \times 10^{4}$ cells $\left./ \mathrm{ml}\right)$, both of which were the two main types of cells isolated by the flow cytometry, were suspended in DMEM/F12 culture medium containing $10 \% \mathrm{FBS}, 20 \mathrm{ng} / \mathrm{ml}$ hepatocyte growth factor (Sigma-Aldrich; Merck KGaA), $5 \mu \mathrm{g} / \mathrm{ml}$ insulin (Sigma-Aldrich; Merck $\mathrm{KGaA}), 10,00,000 \mathrm{U} / 1$ penicillin and streptomycin, and seeded at a density of $1 \times 10^{4}$ cells $/ \mathrm{ml}$. The cultures were passaged after 3 days and harvested following a further 3 days in culture followed by flow cytometric analysis to detect the ratio of SP and NSP cells. BD FACSDiva version 6.1.1 software (BD Biosciences, Franklin Lakes, NJ, USA) was used for analysis.

Cell cycle analysis. Cell cycle analysis was performed using a cell cycle detection kit (Nanjing KeyGen Biotech Co., Ltd., Nanjing, China) according to the manufacturer's protocol. Briefly, the cells were washed with PBS once and centrifuged at $376 \mathrm{~g}$ at $4^{\circ} \mathrm{C}$ for $5 \mathrm{~min}$. The cells $\left(1 \times 10^{6}\right.$ cell $\left./ \mathrm{ml}\right)$ were fixed overnight in $70 \%$ ethanol at $4^{\circ} \mathrm{C}$ followed by washes with PBS. The cells were treated with $500 \mu 1$ PBS containing $50 \mu \mathrm{g} / \mathrm{ml}$ PI, $100 \mu \mathrm{g} / \mathrm{ml}$ RNase A and $0.2 \%$ Triton X-100 at $4^{\circ} \mathrm{C}$ for $30 \mathrm{~min}$ in dark. The stained cells were loaded on a BD Influx cell sorter (BD Biosciences) and the cell cycle was analyzed by BD FACSDiva version 6.1.1 software (BD Biosciences).

Analysis of in vitro clonogenic capacity by soft agar assay. The sorted SP and NSP cells were seeded at a density of 100 cells/well in 24-well plates with soft agar. The upper agar layer consisted of $0.35 \%$ agarose in DMEM supplemented with $10 \%$ FBS, and the base layer was made up of $0.6 \%$ agarose. A total of $500 \mu \mathrm{l}$ fresh medium was added to the cells twice a week. After 3 weeks of culture at $37^{\circ} \mathrm{C}$, the colonies were stained with $0.5 \mathrm{ml} 0.005 \%$ crystal violet for $1 \mathrm{~h}$. Colonies were examined under phase contrast microscopy (IX50; Olympus Corporation, Tokyo, Japan). Colonies with a diameter $>75 \mu \mathrm{m}$ or containing $>50$ cells were counted. The colony formation rate was calculated as follows: Colony formation rate $(\%)=$ number of colonies/number of seeded cells $\mathrm{x} 100$. Experiments were conducted in triplicate.

Tumorigenicity assay for SP and NSP cells. The sorted SP and NSP cells were washed twice in PBS. A total of $1 \times 10^{3}, 1 \times 10^{4}$ and $1 \times 10^{5} \mathrm{SP}$ and NSP cells were resuspended in a mixture of DMEM/F12 (total volume, $200 \mu \mathrm{l}$; ratio, 1:1) and Matrigel. NOD-SCID mice were assigned into three groups ( $\mathrm{n}=5$ for each group) and received subcutaneous injection of different number of cells. The SP cells were injected into the right forelimb, and NSP cells were injected into the left forelimb. The mice were examined daily, and the time points at which tumors appeared were recorded. The tumor formation rate was determined by the following equation: The tumor formation rate $=($ The number of animals developed tumor/The overall number of animals inoculated with tumor cells) x100\%. The mice were sacrificed 12 weeks following inoculation and, the tumor tissues were collected, weighed, fixed in $10 \%$ formalin for 3 days at room temperature and sectioned (3- $\mu \mathrm{m}$ thick). Subsequently, hematoxylin and eosin staining and histological analysis were performed.
For hematoxylin and eosin staining, the paraffin sections were stained with alum haematoxylin, rinsed in running tap water, differentiated with $0.3 \%$ acid alcohol and subsequently rinsed in running tap water. The sections were stained with eosin for 2 min, dehydrated, cleaned and mounted.

RNA preparation and reverse transcription quantitative polymerase chain reaction (RT-qPCR) analysis. Total RNA was extracted with RNeasy Mini kit (Qiagen GmbH, Hilden, Germany) according to the manufacturer's protocol. cDNA synthesis was performed with 500 ng RNA using First-Strand cDNA Synthesis Supermix (TransGen, Beijing, China). Negative controls were included, using distilled water instead of samples. qPCR was performed using FastStart Universal SYBR Green Master (Rox) kit (Roche Diagnostics GmbH, Mannheim, Germany) and run on a StepOnePlus Real-Time PCR system (Applied Biosystems; Thermo Fisher Scientific, Inc., Waltham, MA, USA). The specific primer sequences for the human $A B C G 2$ gene and an endogenous control $\beta$-actin are as follows: ABCG2 forward, 5'-TTCGGCTTGCAACAA CTATG-3' and reverse, 5'-TCCAGACACACCACGGATAA-3'; $\beta$-actin forward, 5'-ATGGTGGGTATGGGTCAGAA-3' and reverse, 5'-CGGAGCTCGTTGTAGAAGGT-3'. The PCR reactions included pre-incubation at $95^{\circ} \mathrm{C}$ for $5 \mathrm{~min}$ and 45 cycles of denaturation at $95^{\circ} \mathrm{C}$ for $15 \mathrm{sec}$, annealing at $60^{\circ} \mathrm{C}$ for $45 \mathrm{sec}$ and extension at $72^{\circ} \mathrm{C}$ for $30 \mathrm{sec}$. Data were calculated and normalized to the reference gene $\beta$-actin using the $2^{-\Delta \Delta C q}$ method (13). Experiments were conducted in triplicate.

Statistical analyses. The SPSS software (version 18.0; SPSS, Inc., Chicago, IL, USA) was used for statistical analysis, and the differences between groups were assessed by unpaired $\mathrm{t}$-test. $\mathrm{P}<0.05$ was considered to indicate a statistically significant difference.

\section{Results}

Establishment of primary cervical cancer cell culture and tumorigenicity assay for the primary cells. Primary cultures were initiated from multiple cervical tissue specimens of G3 squamous cell carcinomas by explant culture method. One primary culture was successfully established from a poorly differentiated squamous carcinoma. The growth of the primary cells was slow at the early stages. The cultured cells reached $80 \%$ confluence in 8-10 days and $100 \%$ confluence in 10-15 days. Initially, the primary cultures were sub-cultured at 8-10 days. Following 8 passages, the cells appeared to be bigger and grew actively with a passaging duration of 6-7 days. Thereafter, these cultured primary cells maintained stable features through subsequent passages although variable cell size and morphology including polygonal, oval and mosaic-like cell appearance was observed. These cells exhibited features including multi-layer growth, nuclear atypia, large nucleus-to-cytoplasm ratio in the majority of cells, strong nuclear staining and prominent pathological mitosis (Fig. 1A). Tumorigenicity assay indicated that lumps with a mean diameter of $4 \mathrm{~mm}$ were observed at injection sites in nude mice $(\mathrm{n}=5) 6$ to 7 days following subcutaneous inoculation of $1 \times 10^{6}$ primary cancer cells at passage 10 . The lumps gradually increased in size with time. The mice were sacrificed 
8 weeks following inoculation, and the mean weight of the tumors was $16.54 \mathrm{~g}$ (Fig. 1B, Table I). Hematoxylin and eosin staining indicated that the tumors are poorly differentiated squamous-cell carcinomas, which is consistent with the cell pathology of the tumors from the original patients (Fig. 1C). Similar to primary tumors, these tumors also expressed the cervical cancer specific marker, p63 (Fig. 1D and E).

Proportion of SP cells in primary cervical cancer cell culture. Primary cancer cells at passage 10 were stained with Hoechst 33342 and analyzed by FACS. The two-dimensional dot plot is shown in Fig. 2 with Hoechst red fluorescence plotted on the $\mathrm{x}$-axis and Hoechst blue fluorescence plotted on the y-axis. The majority of cells were positively stained and gathered in the central and upper right area of the plot (Fig. 2A). In the non-verapamil treated group, a small portion of cells with low Hoechst staining gathered in the lower left corner of the plot (blue), which represented the SP cells. The SP cells show increased efflux of Hoechst 33342 compared with NSP. The proportion of SP cells in the primary cervical cancer culture was $4.73 \%$. Therefore, the majority of the cells that are positive for Hoechst 33342 staining were NSP cells (Fig. 2A). When the $\mathrm{ABC}$ transporter protein inhibitor verapamil was added, the proportion of SP cells with low Hoechst staining was markedly decreased $(<1 \%)$ due to the block of Hoechst efflux (Fig. 2B). Consequently, the majority of the cells were clustered in the upper right quadrant of the plot, which is similar to Fig. 2A.

Cell cycle analyses of SP and NSP cells. The cell cycle distribution patterns of SP and NSP cells are shown in Fig. 3A, B and Table II. $4.17 \%$ of the SP cells and $8.32 \%$ of the NSP cells were in S phase. $95.65 \%$ of the SP cells and $87.43 \%$ of the NSP cells were in G1. $0.18 \%$ of the SP cells and $4.24 \%$ of the NSP cells were in G1. $0.18 \%$ of the SP cells and $4.25 \%$ of the NSP cells were in $\mathrm{G} 2$.

Proliferation potency and self-renewal capacity of SP and NSP cells. Flow cytometric analyses revealed that after 6 days of culture, the proportion of SP cells in the SP cell culture was 6.8 and $1.7 \%$ in the NSP cell culture (Fig. 3C and D).

Differential expression of ABCG2 in SP and NSP cells. $A B C G 2$ encodes an ABC (ATP-binding cassette) family transporter, which pumps out cytotoxic agents and fluorescent dyes including Hoechst 33342 from cells (14). Marked expression of $A B C G 2$ was detected in the SP cells but not in the NSP cells by RT-qPCR (Fig. 4).

Clonogenic capacity of SP cells and NSP cells from primary cervical cancer cells. The clonogenic capacity of SP cells and NSP cells was assessed by soft agar assay with an inoculation density of 100 cells/well. Compared to the NSP cells, the SP cells formed an increased number of colonies ( $>50$ clones/well) with a greater number of cells in each colony ( $>50$ cells/clone) (Fig. 5A). The colony formation rate of SP cells was $60 \%$. By contrast, the NSP cells formed few colonies ( $<20$ clones/well) with a $20 \%$ clone formation rate (Fig. 5B). There was a statistically significant difference between the clone formation rate of SP cells and NSP cells $(\mathrm{P}<0.05)$.
Table I. Cervical cancer tumor formation.

Tumor size,

Mouse no. $(\mathrm{n}=5) \quad$ length $\mathrm{x}$ width $(\mathrm{cm}) \quad$ Tumor weight $(\mathrm{g})$

\begin{tabular}{lll}
\hline 1 & $4.1 \times 2.9$ & 17.2 \\
2 & $4.7 \times 2.8$ & 18.3 \\
3 & $3.9 \times 3.3$ & 15.1 \\
4 & $3.5 \times 2.7$ & 15.2 \\
5 & $4.7 \times 2.5$ & 16.9 \\
\hline
\end{tabular}

A total of $1 \times 10^{6}$ cells were injected into each nude mouse. The size and weight of tumors was measured 8 weeks following inoculation.

Table II. Cell cycle analysis of primary cervical cancer cells, SP cells and NSP cells.

\begin{tabular}{lcccc}
\hline Groups & $\begin{array}{c}\mathrm{G} 0 / \mathrm{G} 1 \\
(\%)\end{array}$ & $\begin{array}{c}\mathrm{G} 2 \\
(\%)\end{array}$ & $\begin{array}{c}\mathrm{S} \\
(\%)\end{array}$ & $\begin{array}{c}\mathrm{G} 2 \text { and S } \\
(\%)\end{array}$ \\
\hline $\begin{array}{l}\text { Primary cervical } \\
\text { cancer cell }\end{array}$ & 68.87 & 19.2 & 11.93 & 31.13 \\
SP & & & & \\
NSP & 95.65 & 0.18 & 4.17 & 4.25 \\
& 87.43 & 4.25 & 8.32 & 12.57 \\
\hline
\end{tabular}

SP, side population; NSP, non-side population.

Tumorigenicity of SP and NSP cells from primary cervical cancer cells. The tumorigenicity of SP and NSP cells was assessed by subcutaneous inoculation of cells into NOD-SCID mice at different cell numbers $\left(1 \times 10^{3}, 1 \times 10^{4}\right.$ and $1 \times 10^{5}$ cells/mouse). The results show that inoculation of SP and NSP cells was able to induce transplantable tumors in NOD-SCID mice, but the tumors formation rates and number of cells required for tumors formation was significantly different for the two cell types (Table III). SP cells were able to induce tumors at all three cell numbers, with a $60 \%$ tumor formation rate at the 12 th week for inoculation with $1 \times 10^{3}$ cells (Fig. 6A). NSP cells were only able to induce a low rate of tumor formation ( $20 \%$ at the 12 th week) when injected at a high cell number of $1 \times 10^{5}$ cells (Fig. 6B). Furthermore, tumor formation of NSP cells occurred markedly later compared with SP cells. When inoculated with $1 \times 10^{5}$ cells, tumor nodules appeared at the 4th week for SP cells and the 10th week for NSP cells. The size of tumors generated from SP cells was markedly greater compared with the size of tumors from NSP cells (Fig. 6B). These results suggest that the tumorigenicity of SP cells is $>100$ times compared with NSP cells. The tumorigenicity difference, evaluated based on the number of cells injected and the size of the tumors formed, between the two cell types was statistically significant $(\mathrm{P}<0.05)$.

\section{Discussion}

Compared with cultured cell lines, the cells obtained from primary cultures are considered as better experimental models as these cells undergo fewer biological changes and more 

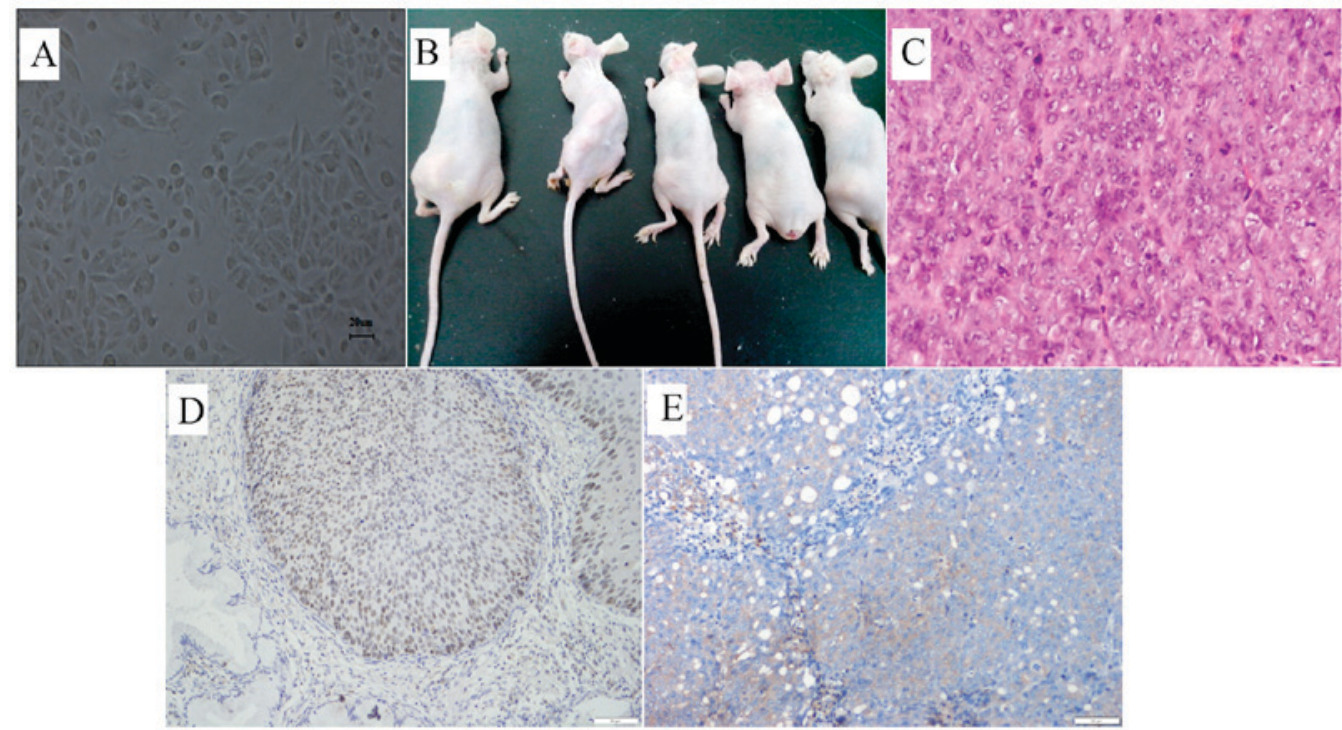

Figure 1. Tumor formation of primary cervical cancer cells in nude mice. (A) Morphology of the primary cervical cancer cells. Scale bar, $20 \mu \mathrm{m}$. (B) Mice with transplanted tumors developed from the primary cervical cancer cells. Animals were sacrificed and images were captured. (C) Hematoxylin and eosin staining of the transplanted tumors developed from the primary cervical cancer cells. Magnification, x40. (D) Positive p63 staining in primary tumor tissue. (E) Positive p63 staining in transplanted tumors. Scale bar, $50 \mu \mathrm{m}$.

A

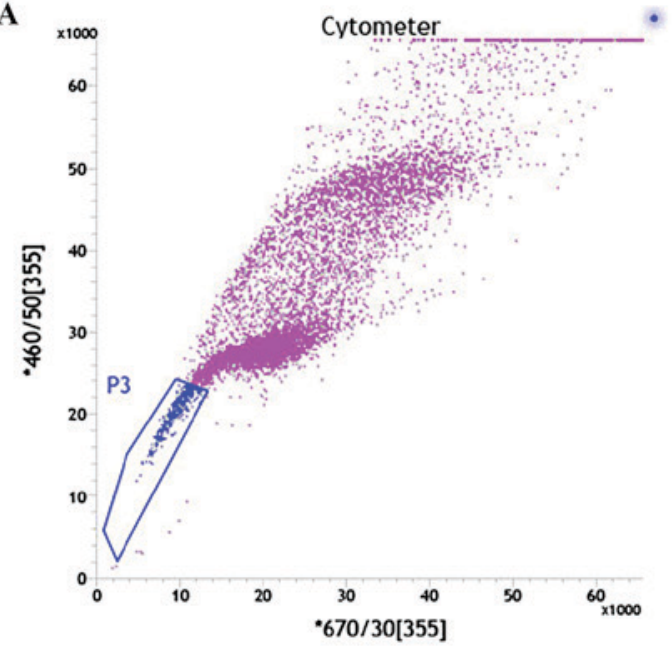

\begin{tabular}{|lrrrrr|r|}
\hline Statistics: Cytometer & & & & & \\
& & & & "610/20... & SSC \\
Populations & Events & \% Total & \% Parent & Moan & Mean \\
\hline All Events & 10,000 & $100.00 \%$ & \#\#\# & 23 & 12,199 \\
\hline P1 & 9,482 & $94.82 \%$ & $94.82 \%$ & 15 & 11,778 \\
\hline P2 & 9,404 & $94.04 \%$ & $99.18 \%$ & 13 & 11,738 \\
\hline P3 & 445 & $4.45 \%$ & $4.73 \%$ & 10 & 9,092 \\
\hline NOT(P3) & 8,959 & $89.59 \%$ & $95.27 \%$ & 14 & 11,870 \\
\hline
\end{tabular}

B

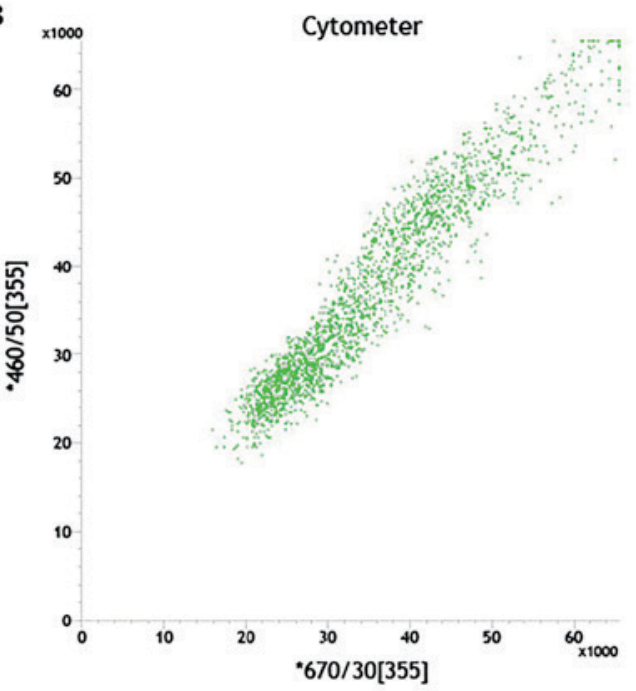

\begin{tabular}{|c|c|c|c|c|c|}
\hline \multicolumn{6}{|c|}{ Statistics: Cytometer } \\
\hline & & & & $* 610 / 20 \ldots$ & SSC \\
\hline Populations & Events & $\%$ Total & \% Parent & Mean & Moan \\
\hline All Events & 10,000 & $100.00 \%$ & \#\#\#\# & 685 & 16,685 \\
\hline P1 & 2,404 & $24.04 \%$ & $24.04 \%$ & 80 & 16,681 \\
\hline $\mathrm{P} 2$ & 2,231 & $22.31 \%$ & $92.80 \%$ & 23 & 16,367 \\
\hline
\end{tabular}

Figure 2. Identification of side population cells by Hoechst 3334 staining and flow cytometry. (A) The majority of the cells (strong fluorescence, NSP cells) were located in the middle and upper right areas of the graph. A small proportion (4.7\%) of cells (SP cells) with a weak fluorescence appeared at the bottom left area of the graph. (B) When $50 \mu \mathrm{mol} / 1$ Verapamil (an inhibitor of the ABCG2 transporter) was added, SP cells disappeared, and all of the cells clustered in the upper right area of the graph. ABCG2, ATP binding cassette subfamily G member 2; NSP, non-side population; SP, side population; SSC, side scatter. 

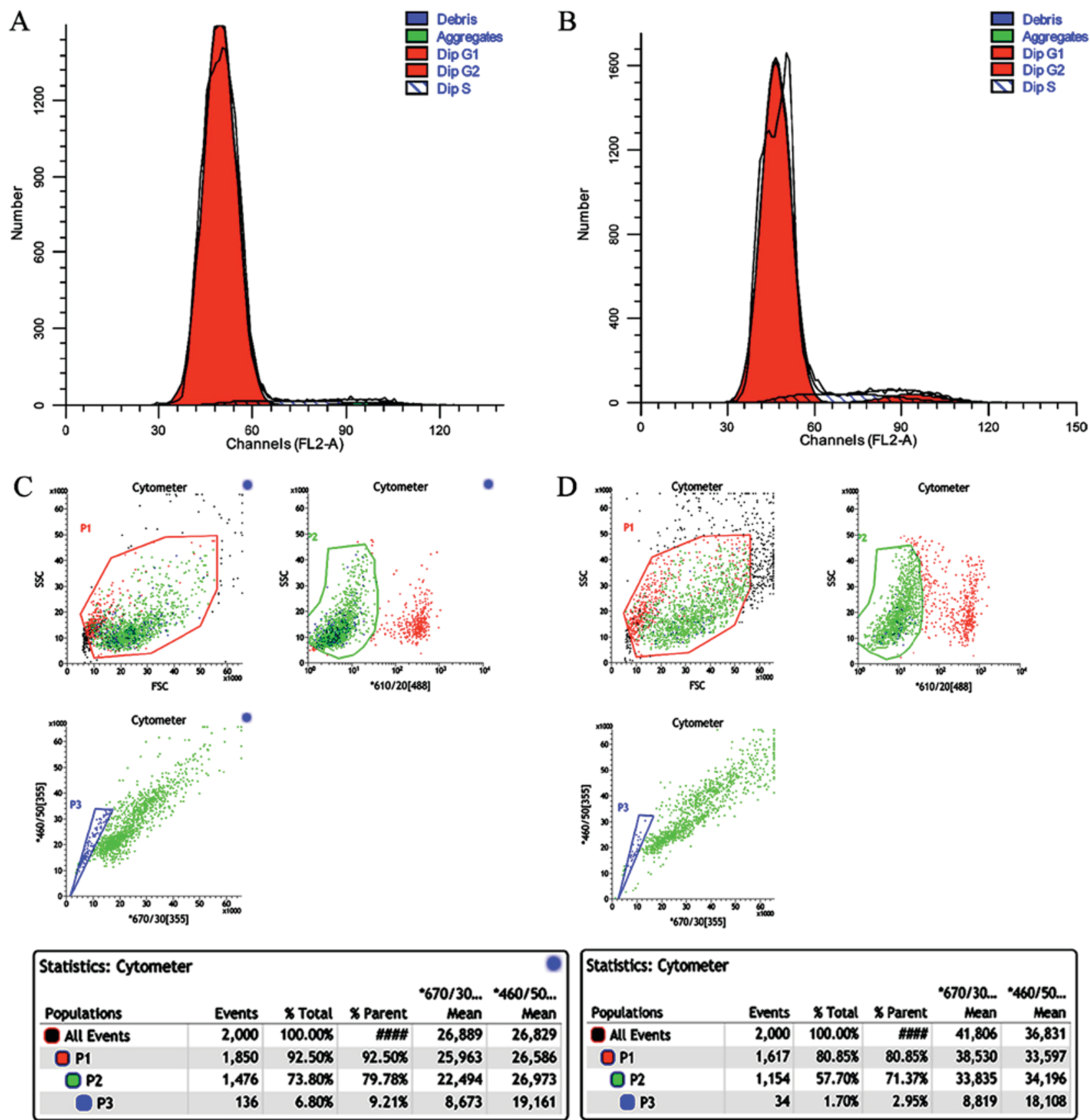

Figure 3. Cell cycle analysis of SP and NSP cells and the proportion of SP cells in SP and NSP cultures. Proportion of (A) SP and (B) NSP cells in G and S. Proportion of SP cells in (C) SP culture (6.8\%) and (D) NSP culture (1.7\%). FSC, forward scatter; NSP, non-side population; SP, side population.

closely resemble the cells within the original in vivo tissue (15). In the present study, only one primary culture was successfully established from a poorly differentiated carcinoma although cultures from multiple cervical tissue specimens of G3 squamous cell carcinoma were initiated. This indicates that choosing poorly differentiated tissues may improve the success rate of primary culture establishment.

SP cells were first identified by Goodell et al (7) when the study of whole bone marrow was conducted using Hoechst 33342 staining. Goodell et al found that a small proportion of cells that had low or negative Hoechst 33342 staining. These cells were called side population because the cells appear in the lower left corner in FACS dot plots $(9-11,16,17)$. The SP cells have low Hoechst 33342 staining as they have a stem cell-like capability to pump out exogenous chemicals including Hoechst 33342. Furthermore, the majority of SP cells have been demonstrated to resemble stem cells in many other aspects in addition to their ability to efflux Hoechst 33342 (18). Side population cells have been observed in a number of tissues, including umbilical cord blood, muscle, bone, lung, liver, pancreas, skin, forebrain, testes, heart, kidney, cornea epithelium edge, prostate, salivary gland and mammary gland (19-29). The proportion of SP cells is very small, representing $0.01-5 \%$ of the total cells $(30,31)$. One study reported that SP cells were identified from well-established cervical cancer cell lines, $\mathrm{HeLa}$ and $\mathrm{SiHa}$, but not from $\mathrm{Ca}$ Ski and C-4 I cell lines (32). The proportion of SP cells was reported to be 1.1 and $0.7 \%$ in $\mathrm{HeLa}$ and $\mathrm{SiHa}$, respectively. Two other studies reported the isolation of a similar proportion of SP cells 


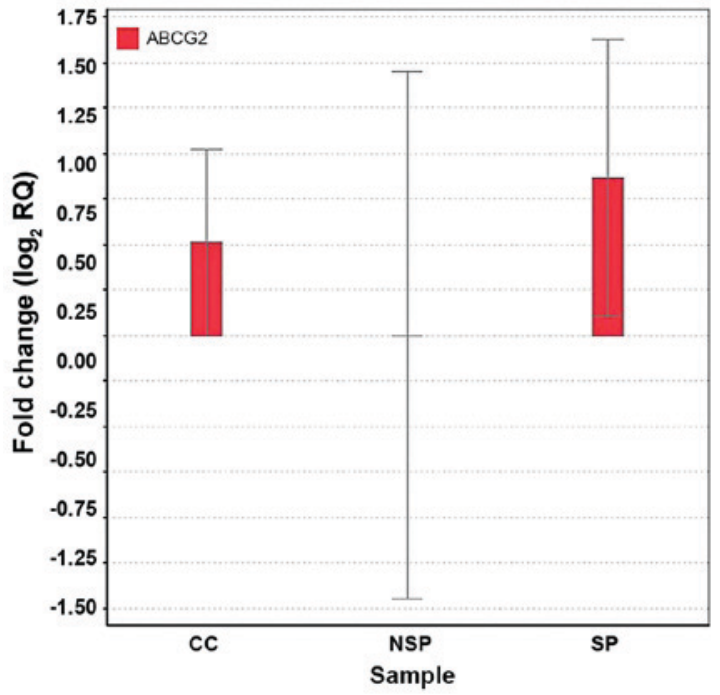

Figure 4. ABCG2 expression in SP and NSP cells determined by reverse transcription quantitative PCR. The relative expression of ABCG2 (normalized to $\beta$-actin) in NSP was set as 1, and the relative expressions of ABCG2 in SP and $\mathrm{CC}$ groups were 1.75 and 1.41, respectively. Data are expressed as the fold change $\left(\log _{2} R Q\right)$ and presented as the mean \pm standard deviation. ABCG2, ATP binding cassette subfamily $\mathrm{G}$ member 2 ; CC, primary cells; RQ, relative quantification. NSP, non-side population; SP, side population.

from the HeLa cell line $(10,11)$. However, the isolation of SP cells from primary cervical cancer cell cultures is rarely reported. In the present study, using FACS with Hoechst 33342 staining, it was demonstrated that SP cells in primary cervical cancer cells accounted for $\sim 4.73 \%$ of total cells. The number of SP cells markedly decreased to $<1 \%$ by the addition of verapamil, which suggests that the majority of SP cells detected in the present study have a stem cell-like capacity to efflux exogenous chemicals. To the best of our knowledge, this is the first report of SP cells isolated from primary cervical cancer cell cultures which may more closely resemble endogenous tissue compared with well-established cancer cell lines including HeLa and $\mathrm{SiHa}$.

Flow cytometric analyses revealed that the proportion of SP cells is relatively high in the primary cells and in the SP culture compared with the proportion in the NSP culture (Figs. 2 and 3). These results suggest that the SP cells have the potency to differentiate into NSP cells, but the NSP cells have negligible differentiation potency. These data also indicated a strong potency for proliferation and self-renewal for SP cells compared with NSP cells. In the present study, the cell cycle analyses demonstrate that SP cells exhibit a lower percentage in the $G_{2}$ and $S$ phases but a higher percentage in the $G_{0} / G_{1}$ phase (Fig. 3A and B; Table II). These data suggested that a larger proportion of SP cells were arrested at G1 checkpoint compared with NSP cells, which resembles the cell cycle distribution of stem cells.

Marked expression of $A B C G 2$ was detected in the SP cells but not in the NSP cells by RT-qPCR (Fig. 4). These results suggested that the ABCG2 may be used as a marker to sort SP cells from cervical cancer cells and to distinguish between SP and NSP cells.

Soft agar assay is an important in vitro method to measure colony formation rate for cancer cells and has been widely used in cancer research for quantifying malignant transformation of cells $(33,34)$. This assay has also been used to

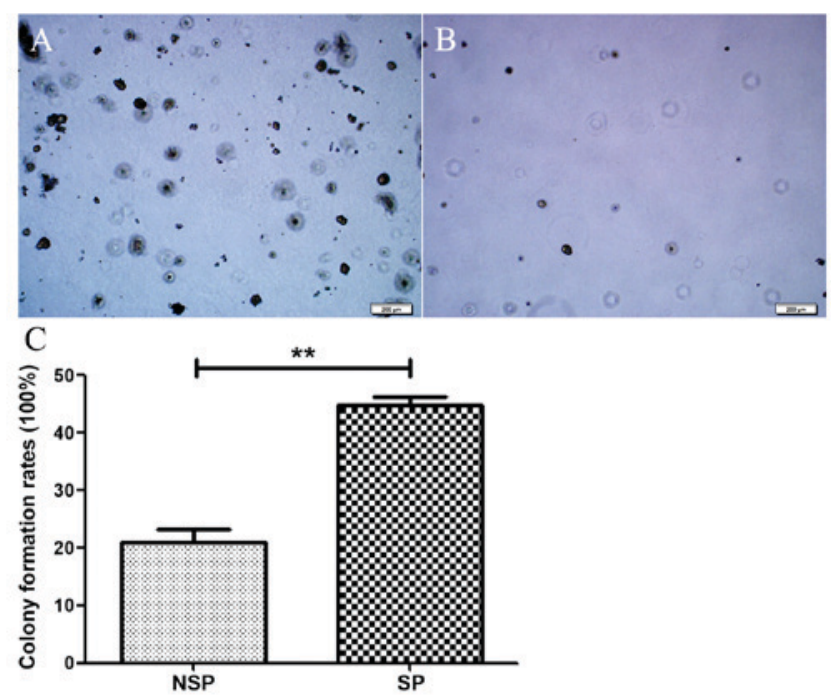

Figure 5. Soft agar assay of SP cells and NSP cells isolated from primary cervical cancer cells. (A) Representative image of colonies formed from SP cells. (B) Representative images of colonies formed from NSP cells. (C) Average colony formation rate. ${ }^{* *} \mathrm{P}<0.01$. SP, side population; NSP, non-side population.

screen and investigate the biological properties of stem cells and progenitor cells (35). It has been previously shown that cells with better colony formation capacity exhibit strong tumorigenicity and metastatic features when tested in animal models and also resemble cancer stem cells in numerous other features (36). In the present study, soft agar assays indicated that the SP cells from primary cervical cancer cell culture presented an increased colony formation capacity compared with NSP cells from the same culture (Fig. 5). These findings from in vitro assays were validated in in vivo tumorigenicity experiments (Fig. 6 and Table III).

As observed for cancer stem cells isolated from acute myeloid leukemia, breast cancer, glioma and other types of cancer, a characteristic feature of cancer stem cells is their strong tumorigenicity $(37,38)$. According to these and other studies, the tumorigenic capacity of cancer stem cells was reported to be 50-1,000 times compared with non-CSC tumor cells. The general method to determine the tumorigenicity of CSCs is to transplant the purified cancer stem cells in immunodeficient mice followed by assessing the timing of tumor formation and the size of tumors developed. In the present study, the SP and NSP cells from the primary cervical cancer cell culture were inoculated subcutaneously in NOD-SCID mice. The results show that whilst SP and NSP cells demonstrated tumorigenic ability, the minimal number of cells required to generate tumors, the timing of tumor initiation, the ratio of tumor formation and the tumor sizes were significantly different between the two groups (Fig. 6 and Table III). The SP cells were able to induce larger tumors with lower inoculation cell densities at earlier time points compared with NSP cells. Overall, the tumorigenicity of SP cells was estimated to be $>100$ times compared with NSP cells. These assays support the hypothesis that the SP cells isolated from primary cervical cancer cell cultures are enriched in CSC-like cells with greater cloning capacity and tumorigenicity when compared with NSP cells. 
Table III. Tumorigenicity of SP and NSP cells from primary cervical cancer cells.

\begin{tabular}{|c|c|c|c|c|c|c|}
\hline \multirow[b]{2}{*}{ Parameters } & \multicolumn{3}{|c|}{ SP } & \multicolumn{3}{|c|}{ NSP } \\
\hline & $1 \times 10^{3}$ & $1 \times 10^{4}$ & $1 \times 10^{5}$ & $1 \times 10^{3}$ & $1 \times 10^{4}$ & $1 \times 10^{5}$ \\
\hline Tumor formation rate & $3 / 5$ & $4 / 5$ & $5 / 5$ & $0 / 5$ & $0 / 5$ & $1 / 5$ \\
\hline Average size $\left(\mathrm{cm}^{3}\right)$ & $1.16 \pm 0.12$ & $1.69 \pm 0.17$ & $2.13 \pm 0.22$ & 0 & 0 & 1.81 \\
\hline
\end{tabular}

A total of $1 \times 10^{3}, 1 \times 10^{4}$ and $1 \times 10^{5}$ cells were injected into each mouse. The tumorigenicity was measured 12 weeks following inoculation. A total of 5 mice were used for each group. SP, side population; NSP, non-side population.
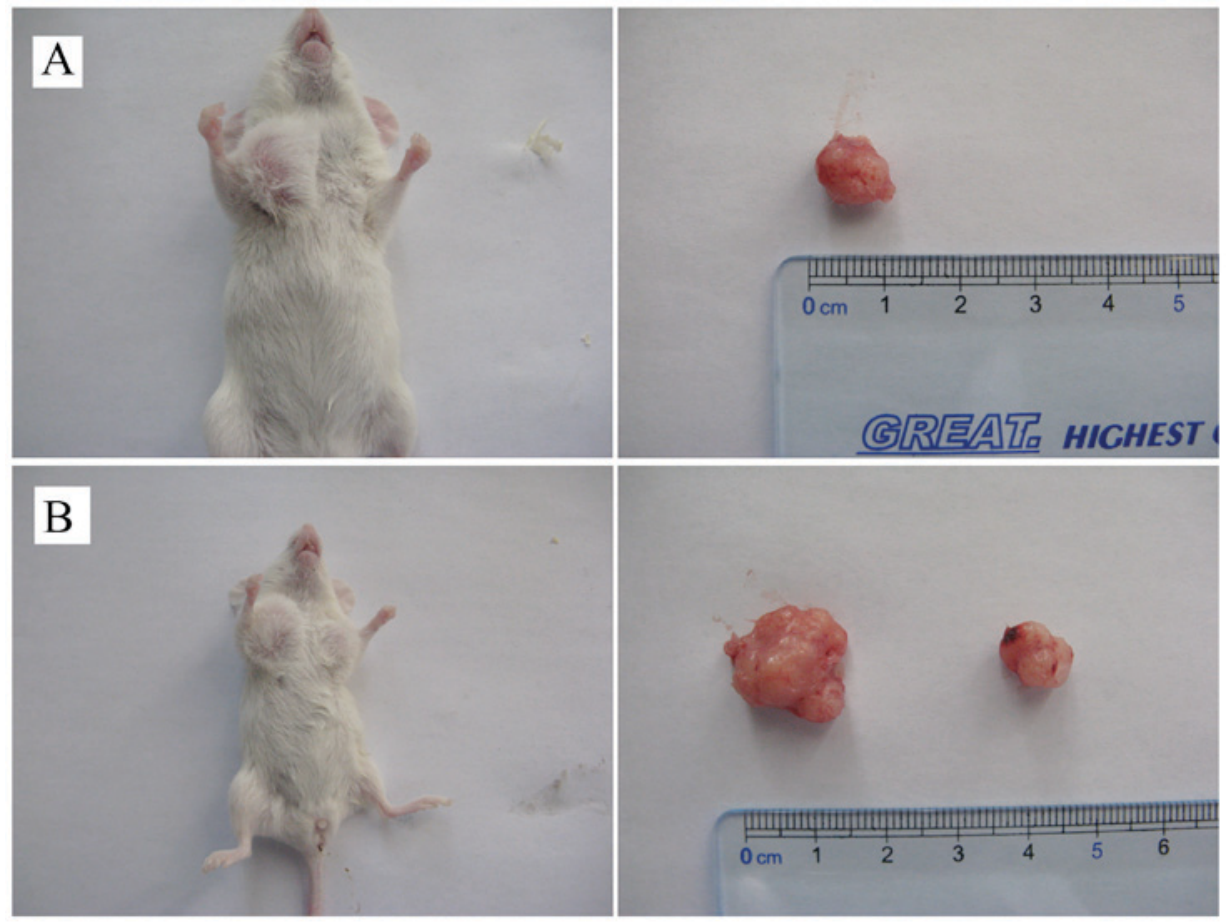

Figure 6. Tumorigenicity assay for SP and NSP cells isolated from patient primary cervical cancer cells. (A) Image of a mouse with a tumor developed following injection of $1 \times 10^{3} \mathrm{SP}$ cells and NSP cells. The SP cells were injected into the right forelimb, and NSP cells were injected into the left forelimbs of NOD-SCID mice. The mice were observed for 12 weeks. (B) Image of a mouse with tumors developed following injection of $1 \times 10^{5} \mathrm{SP}$ cells and NSP cells. Tumors developed in the right (SP cells) and left (NSP cells) forelimbs. NOD-SCID, non-obese diabetic/severe combined immunodeficiency; SP, side population; NSP, non-side population.

The results in the present study on the CSC-like features of SP cells are consistent with observations from other previous studies (39-41). Yu et al reported that the SP cells were able to form spheroids, but the NSP cells failed to generate the typical cell spheres (42). In the present study, the colony formation ratios were 46.82 vs. $12.53 \%$ in the soft agar for SP and NSP cells respectively. Furthermore, $10^{3} \mathrm{SP}$ cells were sufficient to form tumors in NOD-SCID mice. By contrast, $10^{4} \mathrm{NSP}$ cells failed to form tumors. The stem cell features of SP cells from primary cultured human laryngeal squamous cell carcinoma have been previously verified by in vitro and in vivo assays (31). These SP cells also presented increased resistance to chemotherapy, greater xenograft tumorigenicity and higher capacity for proliferation, differentiation and spheroid formation.

To the best of our knowledge, the present study reports the first case of successful isolation of SP cells from a primary cervical cancer cell culture. The isolated SP cells exhibited a greater proliferative and self-renewal potency and increased expression of $A B C G 2$ compared with the NSP cells isolated from the same primary culture. In vitro and in vivo assays demonstrated that these SP cells had markedly increased capacity for colony formation and tumor formation compared with the NSP cells. Although definite molecular stem cell signatures for these SP cells require to be further verified, the present study provides a proof of concept for applying the approach of SP isolation from primary cultures to enrich CSC-like cells from cervical cancer tissues.

\section{Acknowledgments}

The authors of the present study would like to thank Professor Yishu Wang (Department of Pathophysiology, College of Basic Medical Sciences, Jilin University, Changchun, China) for helpful advice with experiments. The authors also would like to acknowledge Professor Yulin Li (Department of Pathophysiology, College of Basic Medical Sciences, Jilin University, 
Changchun, China) for reading an earlier version of the paper and making useful suggestions. Dr Chong Qi and Dr. Gong Peng (Institute of Translational Medicine, The First Hospital of Jilin University, Changchun, China) provided technical support for flow cytometry. The present study was supported by the Project Development Plan of Science and Technology Department of Jilin Province (grant no. 201215046), B Project of Bethune of Jilin University Hospital and the National Natural Science Foundation of China (grant no. 81301884).

\section{References}

1. Reya T, Morrison SJ, Clarke MF and Weissman IL: Stem cells, cancer, and cancer stem cells. Nature 414: 105-111, 2001.

2. Gangemi R, Paleari L, Orengo AM, Cesario A, Chessa L, Ferrini S and Russo P: Cancer stem cells: A new paradigm for understanding tumor growth and progression and drug resistance. Curr Med Chem 16: 1688-1703, 2009.

3. Valent P, Bonnet D, De Maria R, Lapidot T, Copland M, Melo JV, Chomienne C, Ishikawa F, Schuringa JJ, Stassi G, et al: Cancer stem cell definitions and terminology: The devil is in the details. Nat Rev Cancer 12: 767-775, 2012.

4. Bonnet D and Dick JE: Human acute myeloid leukemia is organized as a hierarchy that originates from a primitive hematopoietic cell. Nat Med 3: 730-737, 1997.

5. Al-Hajj M, Wicha MS, Benito-Hernandez A, Morrison SJ and Clarke MF: Prospective identification of tumorigenic breast cancer cells. Proc Natl Acad Sci USA 100: 3983-3988, 2003.

6. Clarke RB, Anderson E, Howell A and Potten CS: Regulation of human breast epithelial stem cells. Cell Prolif 36 (Suppl 1): S45-S58, 2003

7. Goodell MA, Brose K, Paradis G, Conner AS and Mulligan RC: Isolation and functional properties of murine hematopoietic stem cells that are replicating in vivo. J Exp Med 183: 1797-1806, 1996

8. Kondo T, Setoguchi T and Taga T: Persistence of a small subpopulation of cancer stem-like cells in the C6 glioma cell line. Proc Natl Acad Sci USA 101: 781-786, 2004.

9. Patrawala L, Calhoun T, Schneider-Broussard R, Zhou J, Claypool $\mathrm{K}$ and Tang DG: Side population is enriched in tumorigenic stem-like cancer cells, whereas ABCG2+ and ABCG2- cancer cells are similarly tumorigenic. Cancer Res 65: 6207-6219, 2005.

10. Qi W, Zhao C, Zhao L, Liu N, Li X, Yu W and Wei L: Sorting and identification of side population cells in the human cervical cancer cell line HeLa. Cancer Cell Int 14: 3, 2014.

11. Wang K, Zeng J, Luo L, Yang J, Chen J, Li B and Shen K: Identification of a cancer stem cell-like side population in the HeLa human cervical carcinoma cell line. Oncol Lett 6: 1673-1680, 2013.

12. Pecorelli S, Zigliani L and Odicino F: Revised FIGO staging for carcinoma of the cervix. Int J Gynaecol Obstet 105: 107-108, 2009.

13. Livak KJ and Schmittgen TD: Analysis of relative gene expression data using real-time quantitative PCR and the 2(-Delta Delta C(T)) method. Methods 25: 402-408, 2001.

14. Aguilar-Gallardo C, Rutledge EC, Martínez-Arroyo AM, Hidalgo JJ, Domingo S and Simón C: Overcoming challenges of ovarian cancer stem cells: Novel therapeutic approaches. Stem Cell Rev 8: 994-1010, 2012.

15. O Donnell RL, McCormick A, Mukhopadhyay A, Woodhouse LC, Moat M, Grundy A, Dixon M, Kaufman A, Soohoo S, Elattar A, et al: The use of ovarian cancer cells from patients undergoing surgery to generate primary cultures capable of undergoing functional analysis. PLoS One 9: e90604, 2014.

16. Bunting KD: ABC transporters as phenotypic markers and functional regulators of stem cells. Stem Cells 20: 11-20, 2002.

17. Oates JE, Grey BR, Addla SK, Samuel JD, Hart CA, Ramani VA, Brown MD and Clarke NW: Hoechst 33342 side population identification is a conserved and unified mechanism in urological cancers. Stem Cells Dev 18: 1515-1522, 2009.

18. Szotek PP, Pieretti-Vanmarcke R, Masiakos PT, Dinulescu DM, Connolly D, Foster R, Dombkowski D, Preffer F, Maclaughlin DT and Donahoe PK: Ovarian cancer side population defines cells with stem cell-like characteristics and Mullerian Inhibiting Substance responsiveness. Proc Natl Acad Sci USA 103: 11154-11159, 2006.

19. Addla SK, Brown MD, Hart CA, Ramani VA and Clarke NW: Characterization of the Hoechst 33342 side population from normal and malignant human renal epithelial cells. Am J Physiol Renal Physiol 295: F680-F687, 2008.
20. Brown MD, Gilmore PE, Hart CA, Samuel JD, Ramani VA, George NJ and Clarke NW: Characterization of benign and malignant prostate epithelial Hoechst 33342 side populations. Prostate 67: 1384-1396, 2007.

21. Ergen AV, Jeong M, Lin KK, Challen GA and Goodell MA: Isolation and characterization of mouse side population cells. Methods Mol Biol 946: 151-162, 2013.

22. Foster BA, Gangavarapu KJ, Mathew G, Azabdaftari G, Morrison CD, Miller A and Huss WJ: Human prostate side population cells demonstrate stem cell properties in recombination with urogenital sinus mesenchyme. PLoS One 8: e55062, 2013.

23. Habich A, Jurga M, Markiewicz I, Lukomska B, Bany-Laszewicz U and Domanska-Janik K: Early appearance of stem/progenitor cells with neural-like characteristics in human cord blood mononuclear fraction cultured in vitro. Exp Hematol 34: 914-925, 2006.

24. Luth ES, Jun SJ, Wessen MK, Liadaki K, Gussoni E and Kunkel LM: Bone marrow side population cells are enriched for progenitors capable of myogenic differentiation. J Cell Sci 121: 1426-1434, 2008.

25. Scaldaferri ML, Fera S, Grisanti L, Sanchez M, Stefanini M, De Felici M and Vicini E: Identification of side population cells in mouse primordial germ cells and prenatal testis. Int J Dev Biol 55: 209-214, 2011.

26. Uezumi A, Ojima K, Fukada S, Ikemoto M, Masuda S, Miyagoe-Suzuki Y and Takeda S: Functional heterogeneity of side population cells in skeletal muscle. Biochem Biophys Res Commun 341: 864-873, 2006.

27. Umemoto T, Yamato M, Nishida K, Kohno C, Yang J, Tano Y and Okano T: Rat limbal epithelial side population cells exhibit a distinct expression of stem cell markers that are lacking in side population cells from the central cornea. FEBS Lett 579: 6569-6574, 2005.

28. Yoon J, Choi SC, Park CY, Shim WJ and Lim DS: Cardiac side population cells exhibit endothelial differentiation potential. Exp Mol Med 39: 653-662, 2007.

29. Zhang L, Hu J, Hong TP, Liu YN, Wu YH and Li LS: Monoclonal side population progenitors isolated from human fetal pancreas. Biochem Biophys Res Commun 333: 603-608, 2005.

30. Petriz J: Flow cytometry of the side population (SP). Curr Protoc Cytom Chapter 9: Unit 9.23, 2007.

31. Wu C, Wei Q, Utomo V, Nadesan P, Whetstone H, Kandel R, Wunder JS and Alman BA: Side population cells isolated from mesenchymal neoplasms have tumor initiating potential. Cancer Res 67: 8216-8222, 2007.

32. López J, Poitevin A, Mendoza-Martinez V, Pérez-Plasencia C and Garcia-Carranca A: Cancer-initiating cells derived from established cervical cell lines exhibit stem-cell markers and increased radioresistance. BMC Cancer 12: 48, 2012.

33. Horman SR, To J and Orth AP: An HTS-compatible 3D colony formation assay to identify tumor-specific chemotherapeutics. J Biomol Screen 18: 1298-1308, 2013.

34. Jeng KS, Sheen IS, Jeng WJ, Yu MC, Hsiau HI, Chang FY and Tsai HH: Activation of the sonic hedgehog signaling pathway occurs in the CD133 positive cells of mouse liver cancer Hepa 1-6 cells. Onco Targets Ther 6: 1047-1055, 2013.

35. Zheng X, Chopp M, Lu Y, Buller B and Jiang F: MiR-15b and miR-152 reduce glioma cell invasion and angiogenesis via NRP-2 and MMP-3. Cancer Lett 329: 146-154, 2013.

36. Weinberg RA: The biology of cancer. Garland Science, New York, NY, 2007.

37. Kassem NM: Review article: Cancer stem cells: From identification to eradication. J Egypt Natl Canc Inst 20: 209-215, 2008.

38. Ponti D, Costa A, Zaffaroni N, Pratesi G, Petrangolini G, Coradini D, Pilotti S, Pierotti MA and Daidone MG: Isolation and in vitro propagation of tumorigenic breast cancer cells with stem/progenitor cell properties. Cancer Res 65: 5506-5511, 2005.

39. Hirschmann-Jax C, Foster AE, Wulf GG, Nuchtern JG, Jax TW, Gobel U, Goodell MA and Brenner MK: A distinct 'side population' of cells with high drug efflux capacity in human tumor cells. Proc Natl Acad Sci USA 101: 14228-14233, 2004.

40. Moserle L, Ghisi M, Amadori A and Indraccolo S: Side population and cancer stem cells: Therapeutic implications. Cancer Lett 288: 1-9, 2010.

41. Shaharuddin B, Ahmad S, Ali S and Meeson A: Limbal side population cells: A future treatment for limbal stem cell deficiency. Regen Med 8: 319-331, 2013.

42. Yu D, Jin C, Liu Y, Yang J, Zhao Y, Wang H, Zhao X, Cheng J, Liu X and Liu C: Clinical implications of cancer stem cell-like side population cells in human laryngeal cancer. Tumour Biol 34: 3603-3610, 2013. 